\title{
Gmaver \\ Reproducibility study of the Fabbri et al. 2017 model of the human sinus node action potential
}

PHYSIOME ISSN: 2744-6204 Published 01 Sept 2021

OPEN ACCESS Reproducible Model Edited by Shelley Fong Curated by Weiwei Ai

${ }^{*}$ Corresponding author nima.afshar@auckland.ac.nz

Submitted 11 Aug 2021

Accepted 01 Sept 2021

Citation Afshar and Fabbri. Reproducibility study of the Fabbri et al. 2017 model of the human sinus node action potential. Physiome. doi: $10.36903 /$ physiome.16550526

\author{
Nima Afshar ${ }^{*}$, Alan Fabbri ${ }^{2}$, Stefano Severi ${ }^{2}$, Alan Garny ${ }^{1}$, and David \\ Nickerson ${ }^{1}$ \\ ${ }^{1}$ Auckland Bioengineering Institute, University of Auckland, New Zealand \\ ${ }^{2}$ Computational Physiopathology Unit, Department of Electrical, Electronic and Information \\ Engineering "Guglielmo Marconi", University of Bologna, Cesena, Italy
}

\section{ORIG INAL}

\begin{abstract}
The sinoatrial node (SAN) is the natural pacemaker of the mammalian heart. It has been the subject of several mathematical studies, aimed at reproducing its electrical response under normal sinus rhythms, as well as under various conditions.
\end{abstract}

Such studies were traditionally done using data from rabbit SAN cells. More recently, human SAN cell data have become available, resulting in the publication of a human SAN cell model (Fabbri et al., 2017), along with its CellML version.

Here, we used the CellML file provided by the model authors, together with some SED-ML files and Python scripts that we created to reproduce the main results of the aforementioned modelling study.

Keywords: Physiome, CellML, OpenCOR, reproducibility, action potentials, cardiac electrophysiology, cardiovascular models, ion channels, sinoatrial node

\section{Curated Model Implementation}

http://doi.org/10.36903/physiome.16550526

\section{Primary Publications}

A. Fabbri, M. Fantini, R. Wilders, and S. Severi. Computational analysis of the human sinus node action potential: model development and effects of mutations. The Journal of Physiology, 595 (7):2365-2396, 2017.

\section{Introduction}

The sinoatrial node (SAN) plays an important role in cardiac function and although it has been extensively studied, some of its intrinsic mechanisms are still open for debate. Most of the experimental data used in SAN modelling have been carried out on animals and on rabbits in particular (Lakatta and DiFrancesco, 2009; DiFrancesco, 2010; Lakatta, 2010; Maltsev and Lakatta, 2010; Noble et al., 2010; Verkerk et al., 2007; Himeno et al., 2008; DiFrancesco and Noble, 2012; Lakatta and Maltsev, 2012; Rosen et al., 2012; Monfredi et al., 2013; Yaniv et al., 2013, 2015). This has resulted in the development of comprehensive SAN models (Wilders, 2007). Yet, this body of work can hardly be transposed to humans.

Human SAN action potentials were first recorded by Drouin (1997), followed by Verkerk et al. a decade later (Verkerk et al., 2007, 2013). The first human SAN cell model was developed as a 
proof-of-concept by Seemann et al. (2006). This was followed by the model of Chandler et al. (2009). More recently, Verkerk and Wilders (2015) studied the effect of mutations on human SAN cells, highlighting the need for a human-specific SAN cellular electrophysiology model. Such a model was formulated by Pohl et al. (2016), but its action potential shape does not match that of experimental recordings. Fabbri et al. (2017) addressed this shortcoming by developing their human SAN cell model using available human electrophysiological data.

Fabbri et al. (2017) published a CellML version (Cuellar et al., 2003) of their model on the Physiome Model Repository (Yu et al., 2011). However, the CellML file on its own is not sufficient to reproduce all predictions presented in the primary paper. Some SED-ML files (Waltemath et al., 2011) and Python scripts were therefore created and used with the aforementioned CellML file to reproduce the main results from Fabbri et al. (2017). No modifications were made to the CellML file mathematics or parameters and all the equations and parameters can be found in the original paper.

\section{Model description}

Fabbri et al. (2017) developed a human SAN cell model, based on the rabbit SAN cell model of Severi et al. (2012) and on recent electrophysiological data from human SAN cells. The resulting action potential and calcium transient are in agreement with experimentally recorded values. Mutations associated with sinus node dysfunction were also modelled and their effects on pacing rate agree with clinical observations.

The model was developed in Simulink and simulations performed using MATLAB's ode15s solver (Shampine and Reichelt, 1997). Simulations were run until calcium dynamics reached steady-state. Custom MATLAB (2013a) code was used for automatic optimization and feature extraction. A CellML-encoded version of the model is available at https://models.physiomeproject.org/e/568.

The simulation results presented here were produced using the 2021-07-09 snapshot of OpenCOR (Garny and Hunter, 2015) together with various Python scripts that rely on a SED-ML file to configure (the solver to use, the duration of the simulation, the model parameters to track, etc.) and run a given simulation using the model encoded in CellML. Python scripts are also used to generate the figures using Matplotlib (Hunter, 2007).

\section{Model results}
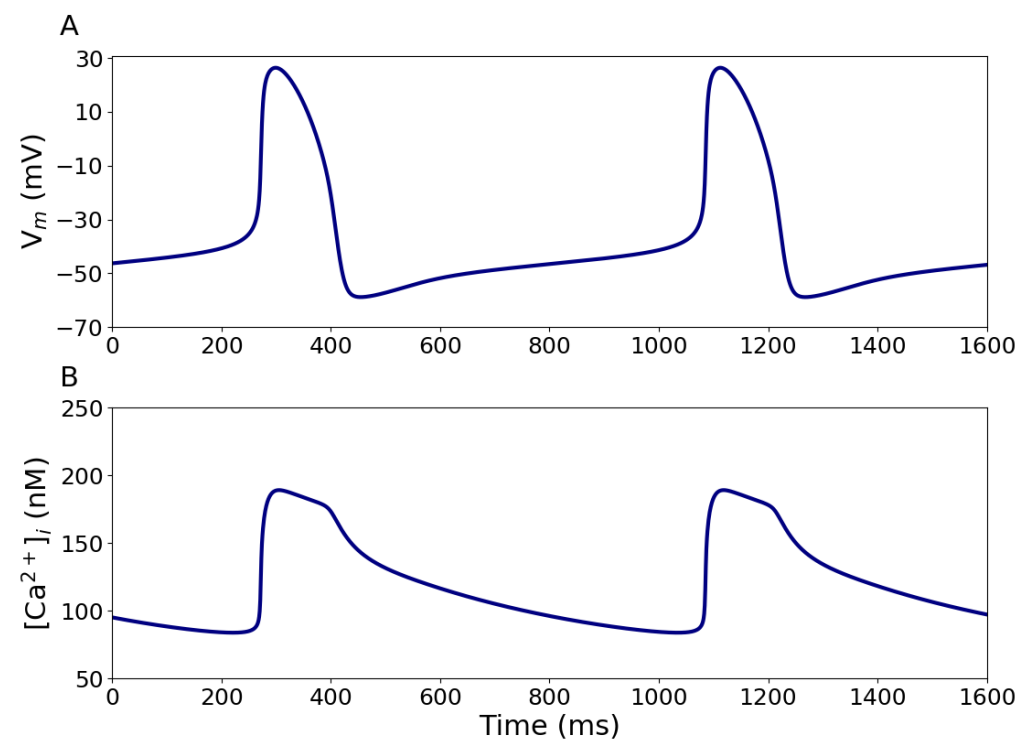

Figure 1. Action potential and intracellular calcium transient of a single human SAN cell.

Simulated action potential (AP; A) and associated calcium transient $\left(\left[\mathrm{Ca}^{2+}\right]_{i} ; \mathrm{B}\right)$ of a single human SAN cell. This figure can be reproduced using Figure1.py. 
Figure 1 shows the action potential (A) and intracellular calcium transient (B), as computed by the model. It reproduces Figure 2 of the primary paper with a cycle length of 814 ms which corresponds to a beating rate of 74 beats $\mathrm{min}^{-1}$. In Figure 2, the time course of the simulated action potential $(A)$ and its underlying components $(B-K)$ are shown, which corresponds to Figure 3 in the primary paper.
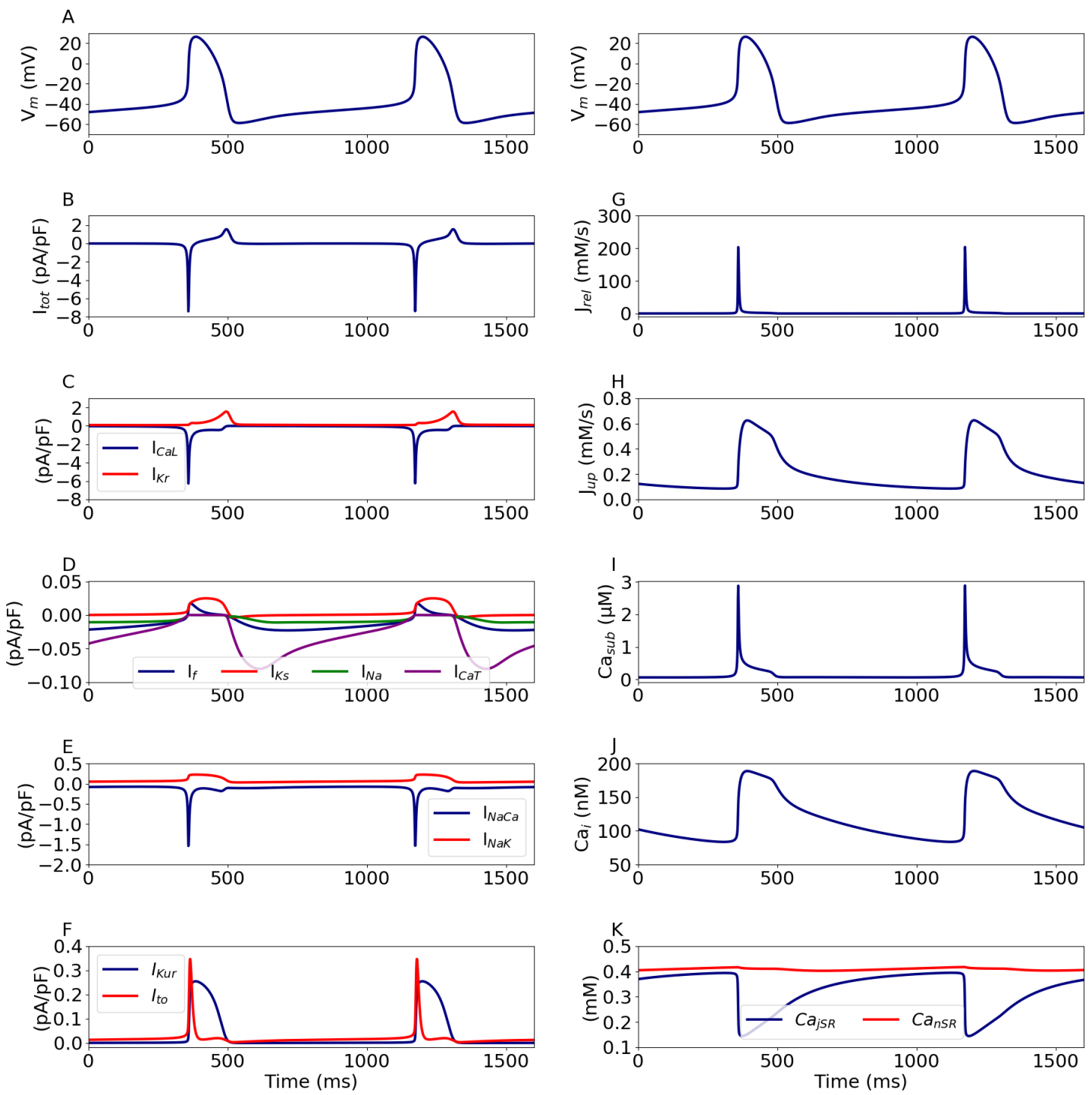

Figure 2. Time course of the simulated action potential and its underlying ionic currents.

Simulated AP (A) and associated currents $(B-F)$, fluxes $(G \& H)$, and calcium concentrations (I-K). The top right panel is a copy of panel $A$ and is included for convenience and to match Figure 3 in the primary paper. This figure can be reproduced using Figure2.py.

Figure 3 shows the membrane potential and its associated currents during diastolic depolarization. The resulting behaviour corresponds to that presented in Figure 4 in the primary paper.

In Figure 4, the model is used to reproduce the results of a progressive block of the funny current, $I_{f}$, to show its effect on the cycle length $(\mathrm{CL})$ and, therefore, on the pacing rate. This corresponds to Figure 7 in the primary paper. 
A
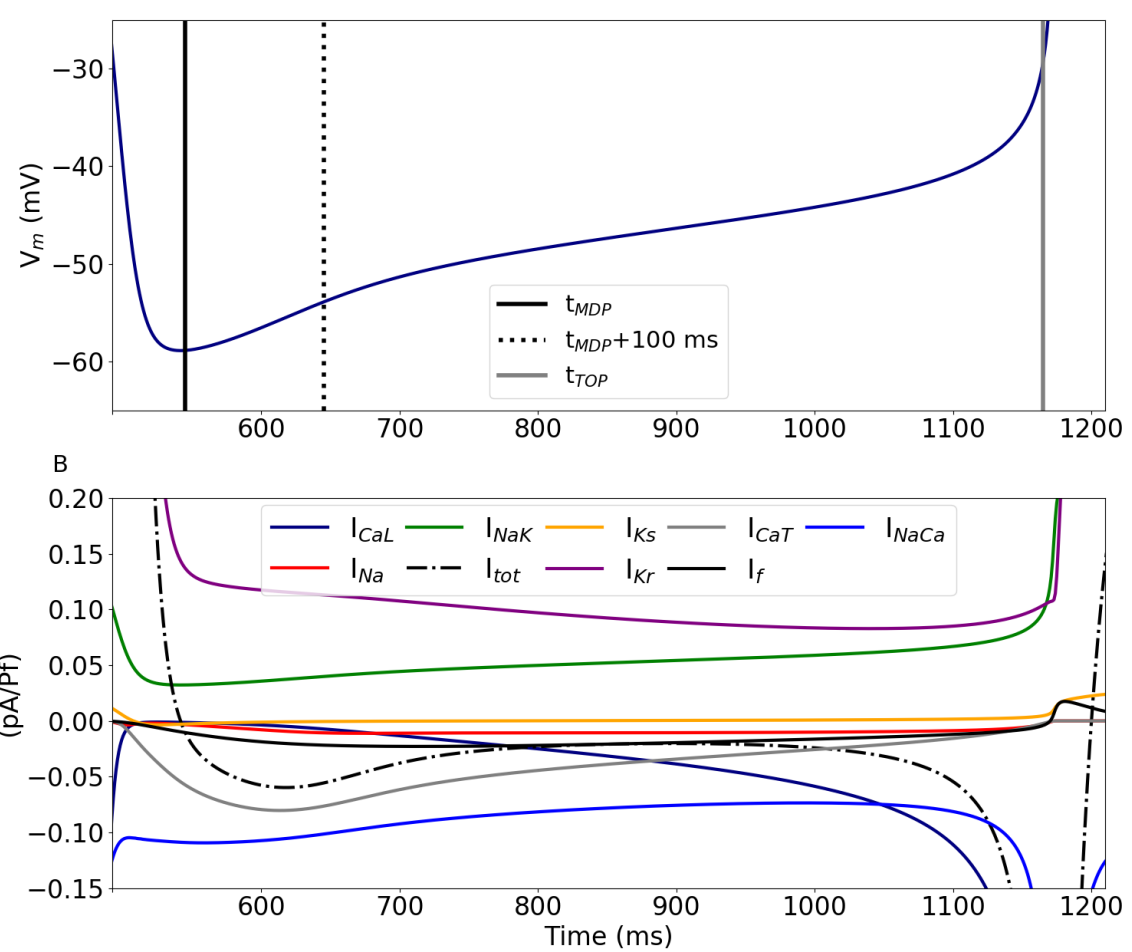

Figure 3. Membrane currents underlying diastolic depolarization.

Simulated AP (A) and associated membrane currents (B). $t_{M D P}$ and $t_{T O P}$ indicate the time at which the membrane potential $\left(V_{m}\right)$ is at its maximum diastolic potential (MDP) and take-off potential, respectively. This figure can be reproduced using Figure3.py.

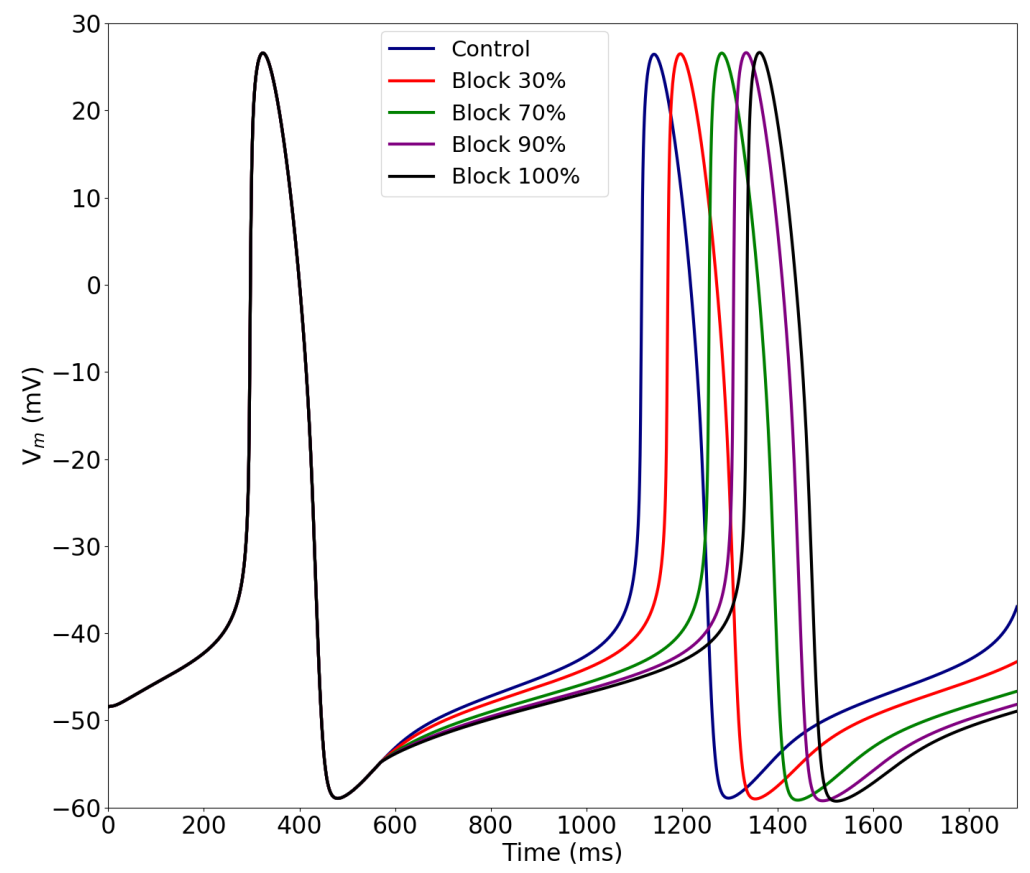

Figure 4. Functional effect of $I_{f}$ block.

Simulated AP under control conditions (CTRL) and upon 30\%, 70\%, $90 \%$ and full block of the funny current $\left(I_{f}\right)$. This figure can be reproduced using Figure4.py. 
Figure 5 shows the effect of a shift in the activation curve of $I_{f}$ on the $C L(A)$, the diastolic depolarization rate over the first $100 \mathrm{~ms}$ of diastolic depolarization $\left(\mathrm{DDR}_{100}\right.$; $\mathrm{B}$ ), the maximum diastolic potential (MDP; C), and the action potential duration at $90 \%$ repolarization $\left(\mathrm{APD}_{90}\right.$; D). This corresponds to Figure 8 in the primary paper. As reported in Fabbri et al. (2017), little variation in $\mathrm{APD}_{90}$ is observed, albeit with slight differences between the Python implementation presented here and the original MATLAB implementation used in the primary paper.
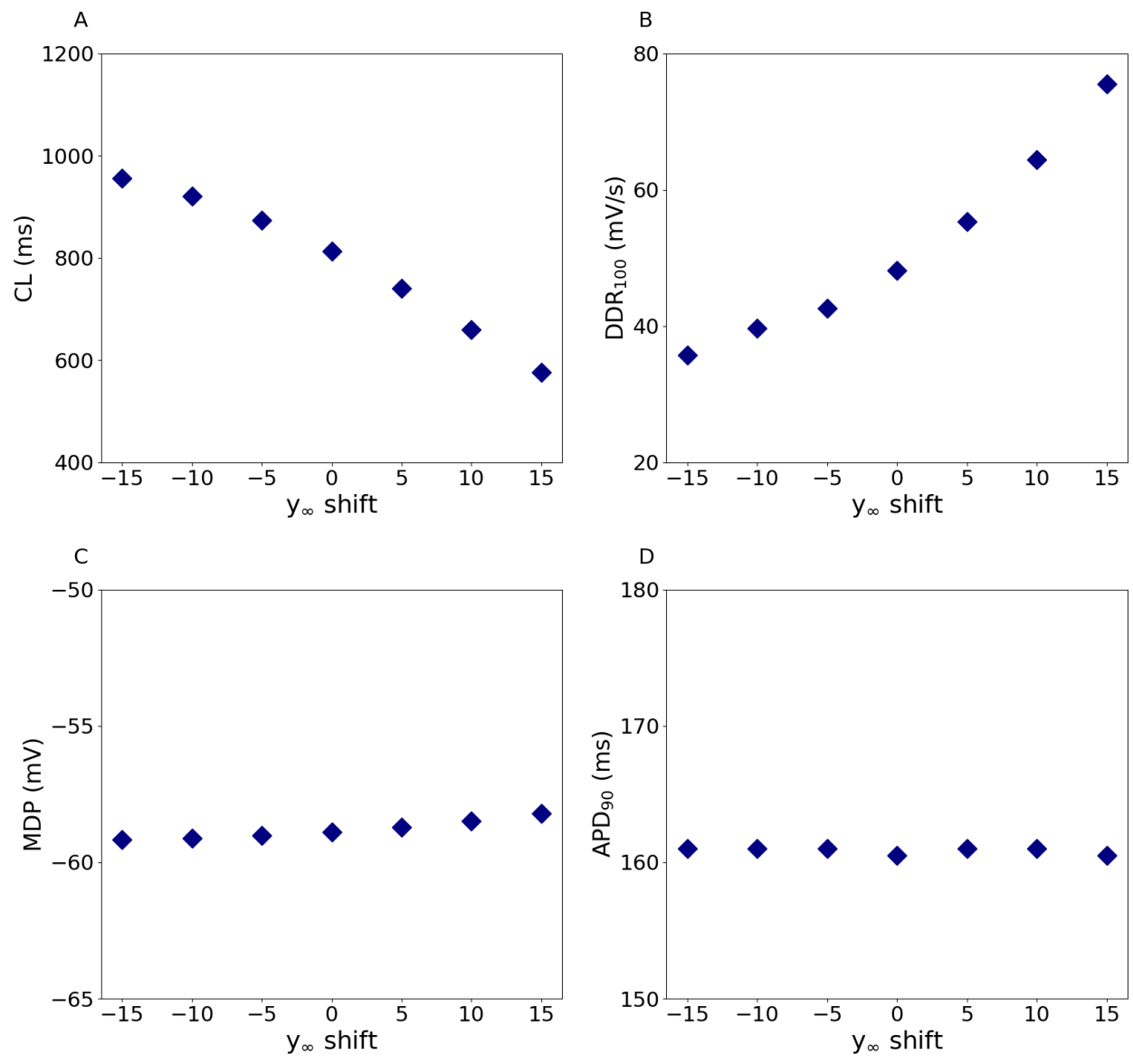

Figure 5. Functional effect of changes in the voltage dependence of $I_{f}$ activation.

Simulations of the effect of -15 to $+15 \mathrm{mV}$ shifts in the voltage dependence of the steady-state activation curve $\left(\mathrm{y}_{\infty}\right)$ of $\mathrm{I}_{f}$ on the cycle length (CL; A), diastolic depolarization rate over the first $100 \mathrm{~ms}$ of diastolic depolarization ( $\left.\mathrm{DDR}_{100} ; \mathrm{B}\right), \mathrm{MDP}(\mathrm{C})$, and action potential duration at $90 \%$ repolarization (APD 90 ; D). This figure can be reproduced using Figure5.py.

The contribution of the sodium-calcium exchanger, $I_{\mathrm{NaCa}}$, to the action potential is illustrated in Figure 6, where a progressive block of $I_{\mathrm{NaCa}}$ was performed. This corresponds to Figure 9 in the primary paper, although the results are slightly different. 

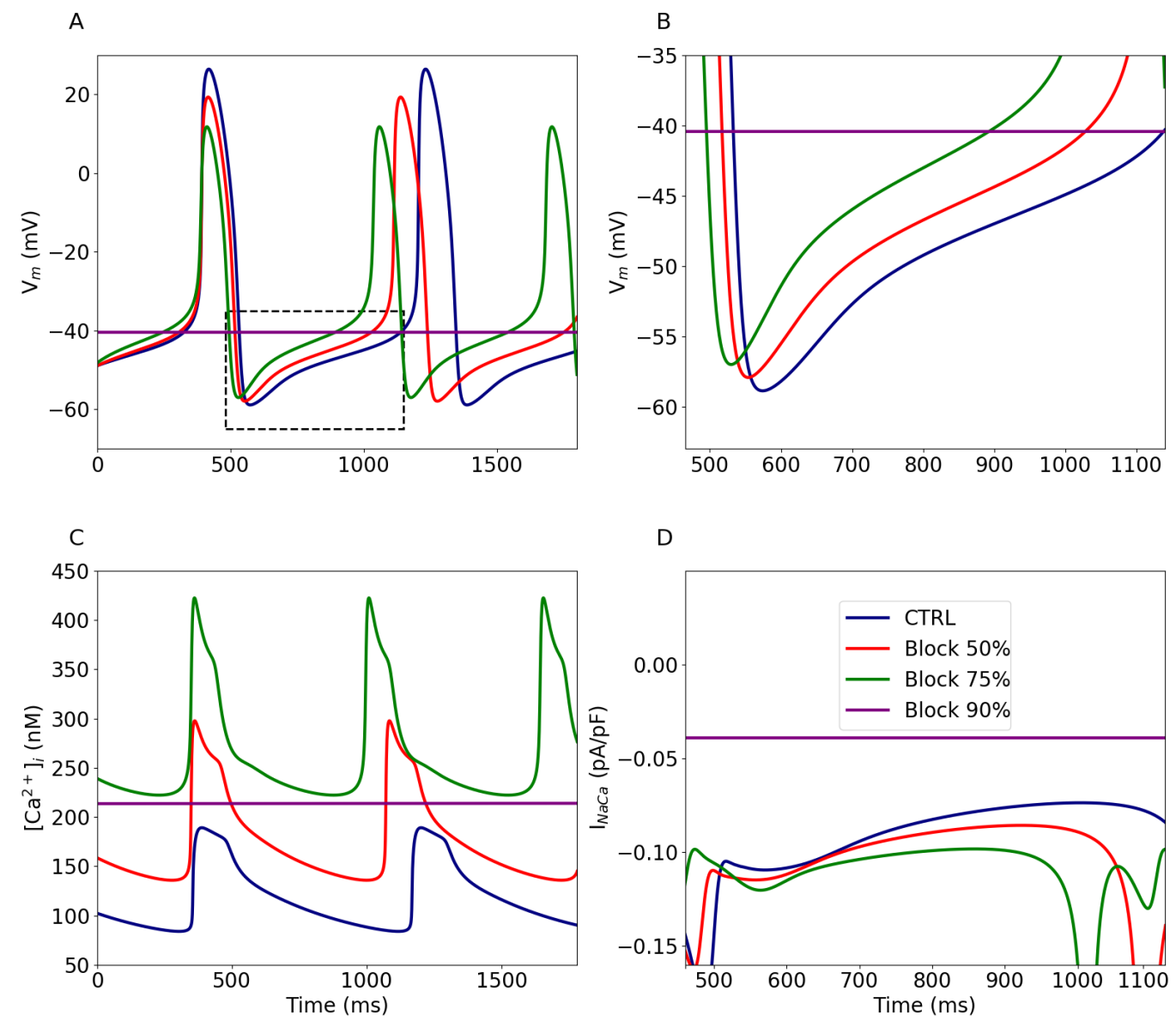

Figure 6. Functional effect of $I_{\mathrm{NaCa}}$ block.

Time course of the simulated $\mathrm{AP}(\mathrm{A})$ and associated $\left[\mathrm{Ca}^{2+}\right]_{\mathrm{i}}(\mathrm{C})$ under control conditions (CTRL) and upon $50 \%, 75 \%$ and $90 \%$ block of $I_{\mathrm{NaCa}}$. Time course of the simulated AP (B) and associated $I_{\mathrm{NaCa}}(\mathrm{D})$ relative to the dashed box of (A). This figure can be reproduced using Figure6.py.

Figure 7 shows the effect of acetylcholine (ACh) and isoprenaline (Iso) on the membrane potential, its net current, the respective target currents for $\mathrm{ACh}$ and Iso, and on the sarco-endoplasmic reticulum $\mathrm{Ca}^{2+}$-ATPase (SERCA) pump uptake rate. This corresponds to Figure 10 in the primary paper. 

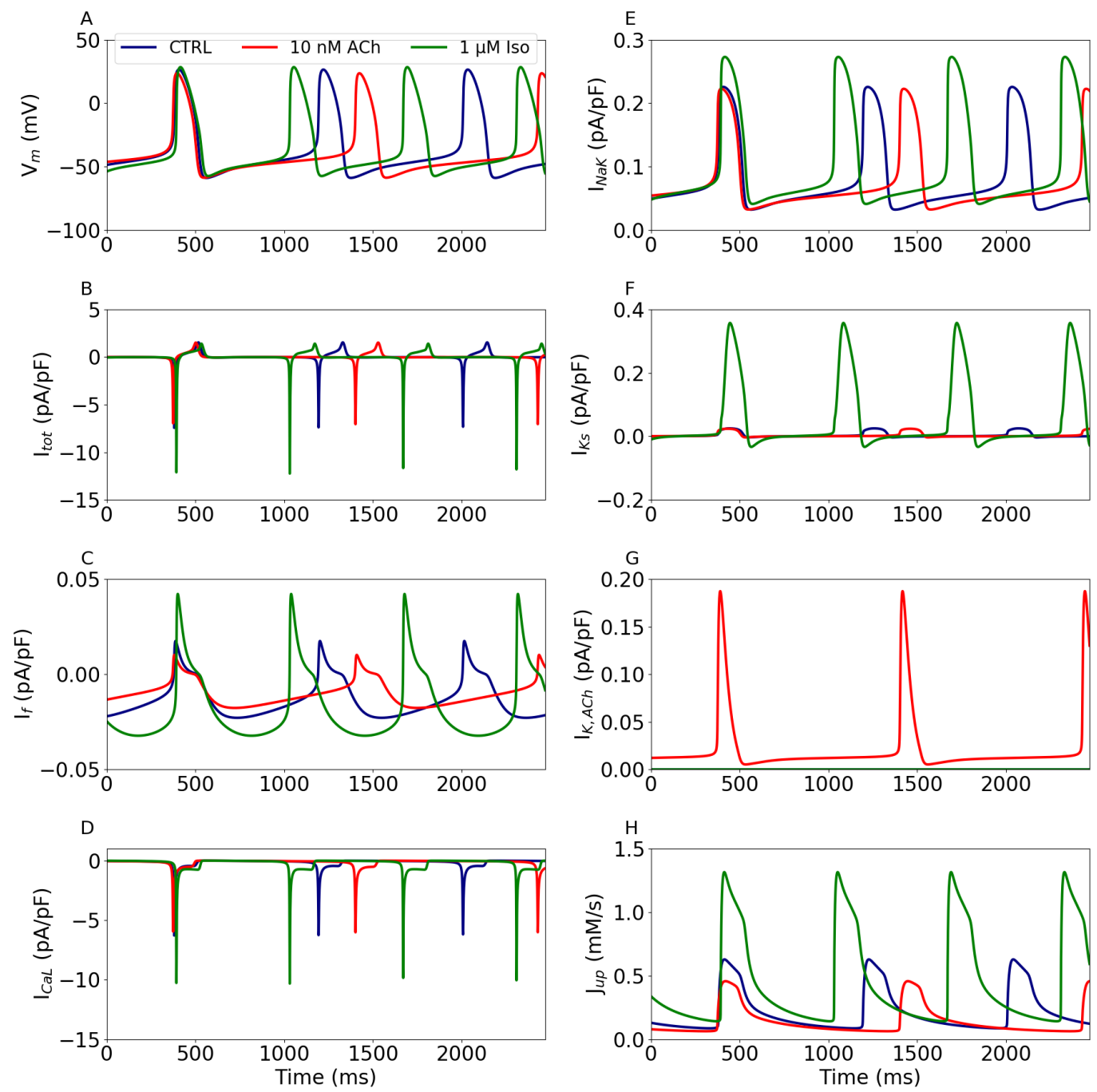

Figure 7. Functional effect of acetylcholine and isoprenaline.

Time course of the membrane potential (A), net current (B), target currents (C-G), and SERCA-pump uptake rate $(\mathrm{H})$ under control conditions (CTRL) and upon administration of $10 \mathrm{nM}$ acetylcholine (ACh) and $1 \mu \mathrm{M}$ isoprenaline (Iso). The targets for $A C h$ are $I_{f}, I_{C a L}, I_{K, A C h}$ and $J_{\text {up }}$ while $I_{f}, I_{C a L}, I_{N a K}, I_{K s}$ and $J_{u p}$ for Iso. Note the differences in ordinate scales. This figure can be reproduced using Figure7.py. 


\section{Discussion}

In this manuscript, we used the CellML version of the human SAN cell model that was developed by Fabbri et al. (2017). Most of the main figures in the primary paper were reproducible using the provided CellML code. In some cases, some Python code was needed and can be found at https://models.physiomeproject.org/workspace/648.

\section{References}

N. Chandler, I. Greener, J. Tellez, S. Inada, H. Musa, P. Molenaar, D. DiFrancesco, M. Baruscotti, R. Longhi, R. Anderson, et al. Molecular architecture of the human sinus node: insights into the function of the cardiac pacemaker. Circulation, 119:1562-1575, 2009.

A. A. Cuellar, C. M. Lloyd, P. F. Nielsen, D. P. Bullivant, D. P. Nickerson, and P. J. Hunter. An overview of CellML 1.1, a biological model description language. Simulation, 79(12):740-747, 2003.

D. DiFrancesco. The role of the funny current in pacemaker activity. Circulation Research, 106(3): 434-446, 2010.

D. DiFrancesco and D. Noble. The funny current has a major pacemaking role in the sinus node. Heart Rhythm, 9(2):299-301, 2012.

E. Drouin. Electrophysiologic properties of the adult human sinus node. Journal of Cardiovascular Electrophysiology, 8(3):254-258, 1997.

A. Fabbri, M. Fantini, R. Wilders, and S. Severi. Computational analysis of the human sinus node action potential: model development and effects of mutations. The Journal of Physiology, 595 (7):2365-2396, 2017.

A. Garny and P. J. Hunter. Opencor: a modular and interoperable approach to computational biology. Frontiers in Physiology, 6:26, 2015.

Y. Himeno, N. Sarai, S. Matsuoka, and A. Noma. Ionic mechanisms underlying the positive chronotropy induced by $\beta 1$-adrenergic stimulation in guinea-pig sinoatrial node cells: a simulation study. The Journal of Physiological Sciences, pages 0801180027-0801180027, 2008.

J. D. Hunter. Matplotlib: A 2d graphics environment. Computing in Science \& Engineering, 9(3): 90-95, 2007. doi: 10.1109/MCSE.2007.55.

E. G. Lakatta. A paradigm shift for the heart's pacemaker. Heart Rhythm, 7(4):559-564, 2010.

E. G. Lakatta and D. DiFrancesco. What keeps us ticking: a funny current, a calcium clock, or both? Journal of Molecular and Cellular Cardiology, 47(2):157-170, 2009.

E. G. Lakatta and V. A. Maltsev. Rebuttal: What if the shoe doesn't fit?"the funny current has a major pacemaking role in the sinus node". Heart Rhythm, 9(3):459-460, 2012.

V. A. Maltsev and E. G. Lakatta. Funny current provides a relatively modest contribution to spontaneous beating rate regulation of human and rabbit sinoatrial node cells. Journal of Molecular and Cellular Cardiology, 48(4):804, 2010.

O. Monfredi, V. A. Maltsev, and E. G. Lakatta. Modern concepts concerning the origin of the heartbeat. Physiology, 28(2):74-92, 2013.

D. Noble, P. J. Noble, and M. Fink. Competing oscillators in cardiac pacemaking: historical background. Circulation Research, 106(12):1791-1797, 2010.

A. Pohl, A. Wachter, N. Hatam, and S. Leonhardt. A computational model of a human single sinoatrial node cell. Biomedical Physics \& Engineering Express, 2(3):035006, 2016.

M. R. Rosen, J. Nargeot, and G. Salama. The case for the funny current and the calcium clock. Heart Rhythm, 9(4):616-618, 2012. 
G. Seemann, C. Höper, F. B. Sachse, O. Dössel, A. V. Holden, and H. Zhang. Heterogeneous three-dimensional anatomical and electrophysiological model of human atria. Philosophical Transactions of the Royal Society A: Mathematical, Physical and Engineering Sciences, 364(1843): 1465-1481, 2006.

S. Severi, M. Fantini, L. A. Charawi, and D. DiFrancesco. An updated computational model of rabbit sinoatrial action potential to investigate the mechanisms of heart rate modulation. The Journal of Physiology, 590(18):4483-4499, 2012.

L. F. Shampine and M. W. Reichelt. The matlab ode suite. SIAM journal on scientific computing, 18 (1):1-22, 1997.

A. O. Verkerk and R. Wilders. Pacemaker activity of the human sinoatrial node: an update on the effects of mutations in hon4 on the hyperpolarization-activated current. International Journal of Molecular Sciences, 16(2):3071-3094, 2015.

A. O. Verkerk, R. Wilders, M. M. van Borren, R. J. Peters, E. Broekhuis, K. Lam, R. Coronel, J. M. de Bakker, and H. L. Tan. Pacemaker current (if) in the human sinoatrial node. European Heart Journal, 28(20):2472-2478, 2007.

A. O. Verkerk, M. M. van Borren, and R. Wilders. Calcium transient and sodium-calcium exchange current in human versus rabbit sinoatrial node pacemaker cells. The Scientific World Journal, 2013, 2013.

D. Waltemath, R. Adams, F. T. Bergmann, M. Hucka, F. Kolpakov, A. K. Miller, I. I. Moraru, D. Nickerson, S. Sahle, J. L. Snoep, and N. Le Novère. Reproducible computational biology experiments with SED-ML-the simulation experiment description markup language. BMC Systems Biology, 5 (1):1-10, 2011.

R. Wilders. Computer modelling of the sinoatrial node. Biopacemaking, pages 121-148, 2007.

Y. Yaniv, S. Sirenko, B. D. Ziman, H. A. Spurgeon, V. A. Maltsev, and E. G. Lakatta. New evidence for coupled clock regulation of the normal automaticity of sinoatrial nodal pacemaker cells: Bradycardic effects of ivabradine are linked to suppression of intracellular ca2+cycling. Journal of Molecular and Cellular Cardiology, 62:80-89, 2013.

Y. Yaniv, E. G. Lakatta, and V. A. Maltsev. From two competing oscillators to one coupled-clock pacemaker cell system. Frontiers in Physiology, 6:28, 2015.

T. Yu, C. M. Lloyd, D. P. Nickerson, M. T. Cooling, A. K. Miller, A. Garny, J. R. Terkildsen, J. Lawson, R. D. Britten, P. J. Hunter, and P. M. F. Nielsen. The Physiome Model Repository 2. Bioinformatics, 27(5):743-744, Jan. 2011. ISSN 1367-4803, 1460-2059. doi: 10.1093/bioinformatics/btq723. URL http://bioinformatics.oxfordjournals.org/content/27/5/743. 
Reproducibility report for: Reproducibility study of the Fabbri et al. 2017 model of the human sinus node action potential.

Submitted to: Physiome

Manuscript number/identifier: S000010

Curation outcome summary: Successfully reproduced all the figures presented in this manuscript.

Box 1: Criteria for repeatability and reproducibility

\section{Model source code provided:}

$\square$ Source code: a standard procedural language is used (e.g. MATLAB, Python, C)

$\square$ There are details/documentation on how the source code was compiled

$\square$ There are details on how to run the code in the provided documentation

$\square$ The initial conditions are provided for each of the simulations

$\square$ Details for creating reported graphical results from the simulation results

Source code: a declarative language is used (e.g. SBML, CellML, NeuroML)

The algorithms used are defined or cited in previous articles

The algorithm parameters are defined

$\square$ Post-processing of the results are described in sufficient detail

\section{$\square$ Executable model provided:}

The model is executable without source (e.g. desktop application, compiled code, online service)

$\square$ There are sufficient details to repeat the required simulation experiments

\section{The model is described mathematically in the article(s):}

$\square$ Equations representing the biological system

$\square$ There are tables or lists of parameter values

$\square$ There are tables or lists of initial conditions

Machine-readable tables of parameter values

Machine-readable tables of initial conditions

The simulation experiments using the model are described mathematically in the article:

$\square$ Integration algorithms used are defined

$\square$ Stochastic algorithms used are defined

$\square$ Random number generator algorithms used are defined

$\square$ Parameter fitting algorithms are defined

The paper indicates how the algorithms yield the desired output 
Box 2: Criteria for accessibility

Model/source code is available at a public repository or researcher's web site

$\square$ Prohibitive license provided

$\square$ Permissive license provided

Open-source license provided

All initial conditions and parameters are provided

All simulation experiments are fully defined (events listed, collection times and measurements specified, algorithms provided, simulator specified, etc.)

Box 3: Rules for Credible practice of Modeling and Simulation ${ }^{a}$

aModel credibility is assessed using the Interagency Modeling and Ananlysis Group conformance rubric: https://www.imagwiki.nibib.nih.gov/content/10-simple-rules-conformance-rubric

Define context clearly: Extensive

Use appropriate data: Extensive

Evaluate within context: Extensive

List limitations explicitly: Insufficient

Use version control: Extensive

Document adequately: Extensive

Conform to standards: Insufficient

Box 4: Evaluation

Model and its simulations could be repeated using provided declarative or procedural code

Model and its simulations could be reproduced 
Summary comments: Model and source code are available in the associated OMEX archive. This was used in our attempt to reproduce the results presented in the paper. We successfully ran the python scripts provided to reproduce Figure 1 - Figure 7 as presented in this manuscript.

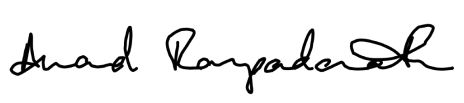

Anand K. Rampadarath ${ }^{1}$, PhD

Curator

Center for Reproducible Biomedical Modeling

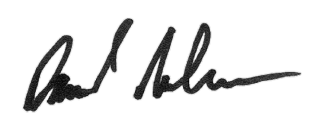

David P. Nickerson, PhD

Curation Service Director

Center for Reproducible Biomedical Modeling

Auckland Bioengineering Institute, University of Auckland

\footnotetext{
1Email: a.rampadarath@auckland.ac.nz
} 